\title{
On Variable Frequency Microwave Processing of Heterogeneous Chip-on-Board Assemblies
}

\author{
T. Tilford ${ }^{1}$, S. Pavuluri ${ }^{2}$, C. Bailey ${ }^{1}$ and M. P. Y. Desmulliez ${ }^{2}$ \\ ${ }^{1}$ School of Computing and Mathematical Sciences, University Of Greenwich, Park Row, \\ Greenwich, London, SE10 9LS, United Kingdom \\ ${ }^{2}$ MicroSystems Engineering Centre (MISEC), School of Engineering \& Physical Sciences, \\ Earl Mountbatten Building, Heriot-Watt University, Edinburgh, EH14 4AS, United Kingdom \\ E-Mail: T. Tilford@gre.ac.uk
}

\begin{abstract}
Variable Frequency Microwave (VFM) processing of heterogeneous chip-on-board assemblies is assessed using a multiphysics modelling approach. The Frequency Agile Microwave Oven Bonding System (FAMOBS) is capable of rapidly processing individual packages on a Chip-On-Board (COB) assembly. This enables each package to be processed in an optimal manner, with temperature ramp rate, maximum temperature and process duration tailored to the specific package, a significant benefit in assemblies containing disparate package types. Such heterogeneous assemblies may contain components such as large power modules alongside smaller modules containing low thermal budget materials with highly disparate processing requirements.

The analysis of two disparate packages has been assessed numerically to determine the applicability of the dual section microwave system to curing heterogeneous devices and to determine the influence of differing processing requirements of optimal process parameters.
\end{abstract}

\section{Introduction}

Semiconductor dies utilised in microelectronics applications generally require encapsulation to protect them from environmental factors such as moisture, ingress of dust or residual humidity. Thermosetting polymer materials, which are often used for this purpose, require heat to initiate or expedite the cure process.

The most common heating system for this process is the convection oven, although approaches such as ultra-violet (UV) and Infrared (IR) heating are also used [reference]. These processes take a relatively long time, often several hours, to bring the encapsulant material up to temperatures which result in a significant cure rate. Convection heating raises the temperature of the gas in contact with the surface of the polymer material and heat energy is transferred into the bulk through thermal conduction. The rate of energy absorption is proportional to the difference in temperature between the package being heated and the ambient temperature in the oven. The temperature profile of the package is therefore asymptotic toward the ambient oven temperature. Electromagnetic energy at infrared or ultraviolet wavelengths will penetrate a small distance (approx. 200-400 $\mathrm{nm}$ for UV and 1-1000 $\mu \mathrm{m}$ for IR) into the load but conduction still limits heat transfer into the bulk material.

An alternative method for polymer curing is the use of Variable Frequency Microwave (VFM) systems, which have been shown to be capable of curing encapsulant materials in substantially shorter times [1]. Electromagnetic energy at microwave frequencies $(1-30 \mathrm{GHz}$.) will generally penetrate several millimetres into the material, leading to much more rapid volumetric heating. The heating rate in this type of system is governed by the operating power and the dielectric properties of the material. Although the dielectric properties of many materials are temperature dependant, the field intensity can be controlled, enabling the heating rate to be varied independently of the temperature of the material. This results in the capability to accurately control the temperature profile, significantly increase heating rates, control how the material will cure, and ultimately increase productivity rates of the packaging of electronic components and their reliability.

A recent innovation in VFM technology is the dual-section VFM system, proposed by Sinclair et al. [2]. In general, VFM systems require the entire board assembly to be placed in an oven and irradiated with electromagnetic energy. The dualsection system is open-ended and is designed to be placed over an individual component. This enables the curing of single components, eliminating thereby unnecessary heating of the remaining components and board assembly, resulting in reduction of thermomechanical stresses and associated reliability improvements. Furthermore, it is possible for the system to be linked to a temperature monitor, providing the capability to accurately control the heating pattern and rate, allowing the process parameters to be optimised for the specific component.

The composition of a dual-section microwave system is depicted schematically in Figure 1. The oven primarily consists of a ceramic (or polymer) block partially filling an open-ended lightweight metal box, with a coaxial cable feed penetrating the ceramic. The system is designed in a way in that electromagnetic fields are induced within the ceramic block and within the section of the oven which is not filled with ceramic but prevents radiation of fields from the open end of the oven.

The system comprises of the oven unit attached to a gantry system placed over a manufacturing line. The oven is attached to the gantry using a tool head which also links the oven to a microwave power source and is able to provide additional tool interchange and temperature sensing functionality. The gantry system enables the oven to be placed over an individual component on a printed circuit board. Once in position, the microwave source is activated to heat the encapsulant and initiate the cure process. Temperature data is fed back to the microwave source enabling accurate control of the induced temperature profile. Once the process is complete, the oven can be placed over the second component and where the process can be repeated. The system is able to use a different temperature profile for each component processed. 


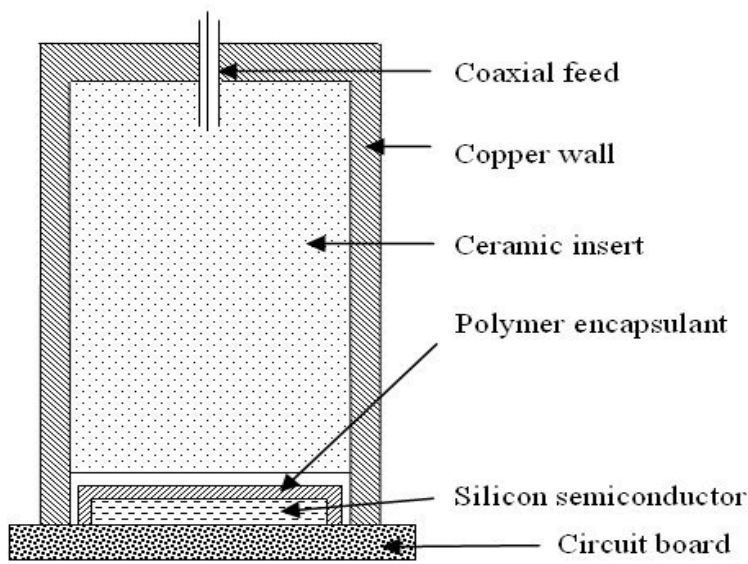

Figure 1 Diagrammatic representation of a dual-section microwave system

This paper aims to numerically assess the curing of encapsulant material in two simplified microelectronics packages with disparate processing requirements. Primary process parameters such as temperature variations, cure rate, degree of cure, and thermomechanical stresses need to be monitored throughout the process, with comparisons indicating benefits and weaknesses of the different heating approaches.

Curing of thermosetting polymer materials is a complex process, with interactions between temperature, curing and stress development. In addition, the analysis of the curing process must also take into account the complex interactions between the component and the electromagnetic fields present due to the microwave energy needed to cure the materials. In order to accurately model the curing process, a holistic approach has been taken, in which the process is not considered to be a sequence of discrete steps, but as a complex coupled system.

Experimental assessment of the key process parameters is extremely complicated, especially if real time/in-situ data are desired. A multiphysics approach to numerical analysis has therefore been adopted to analyse the key process parameters.

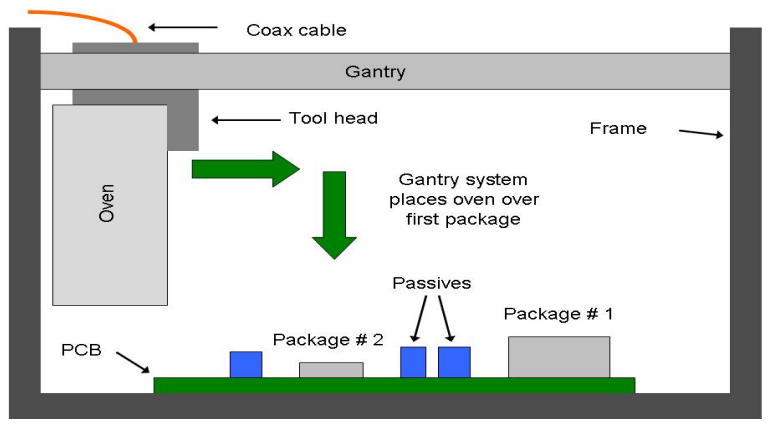

Figure 2 (a) Oven system initially 'parked' to the side of the line

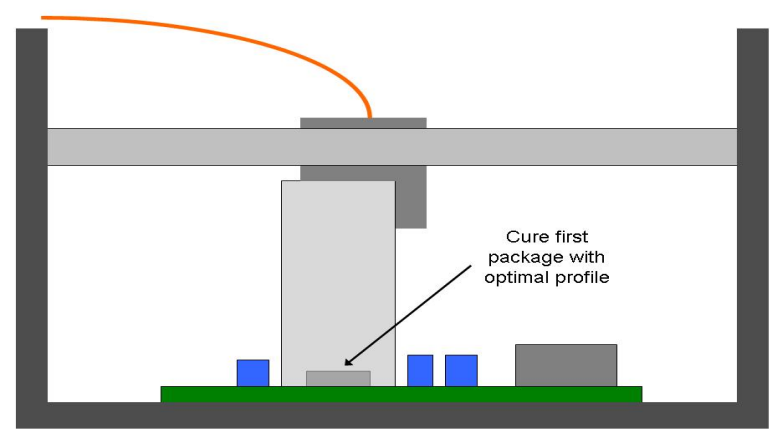

Figure 2 (b) Gantry system moves the oven head over an individual component and the oven is activated to process the component

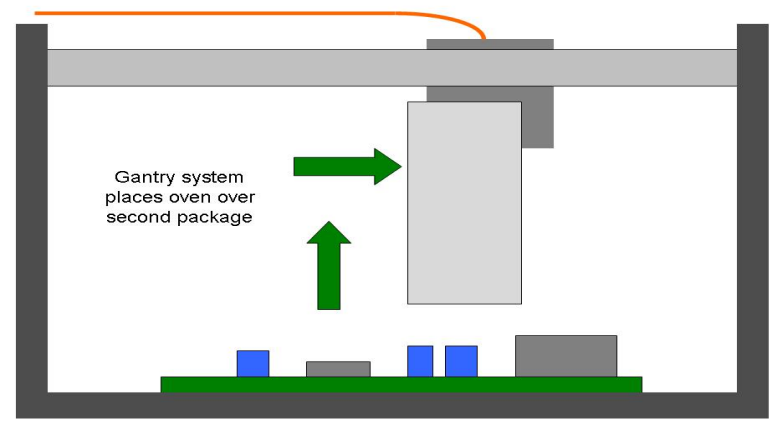

Figure 2 (c) After processing is complete the oven is moved to the next component

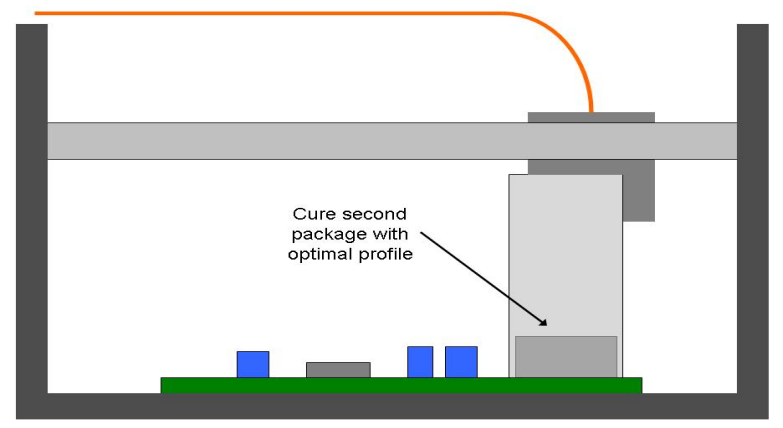

Figure 2 (d) After processing of the second component the oven returns to the starting position

\section{Numerical Model}

A numerical model capable of modelling variable frequency microwave heating has been developed. The model is highly complex, reflecting the intricacy of the process it is required to analyse. The major components of the model are algorithms to solve for the electromagnetic (EM) fields, temperature distribution, cure kinetics and induced thermomechanical stresses. The processes are coupled in a complex manner, as outlined in Figure 3, requiring a holistic approach to analyse the problem

The EM fields induce heating in the package, which expedites the cure process. Thermal expansion and cure shrinkage induce stresses within the package. The temperature 
rise and variation of degree of cure within the material significantly alter the dielectric properties, affecting the EM fields and therefore the heating rate and pattern. Furthermore, the cure reaction is predominantly exothermic, further complicating the relationship between cure rate and temperature.

The numerical model developed adopts a multi-domain FDTD-UFVM approach. The model comprises a Yee scheme [3] Finite Difference Time Domain (FDTD) electromagnetic solver coupled with an unstructured finite volume method (UFVM) multi-physics package [4]. Electromagnetic and thermophysical solutions are obtained within independent numerical domains, with coupling implemented through an inter-domain cross mapping process. The thermophysical analysis mesh is confined to the load material while the electromagnetic mesh occupies the entire oven domain (encompassing the thermophysical domain). This approach enables the FDTD mesh to be varied without requiring modification to the thermophysical mesh. This is of great benefit in cases in which the dielectric properties of the load material (and therefore local propagation wavelength) vary during the heating process. The cross mapping algorithm transfers relevant data between the two domains based on spatial coordinate sampling approach. The model has been shown to provide accurate analysis of complex microwave processing problems $[5,6]$.

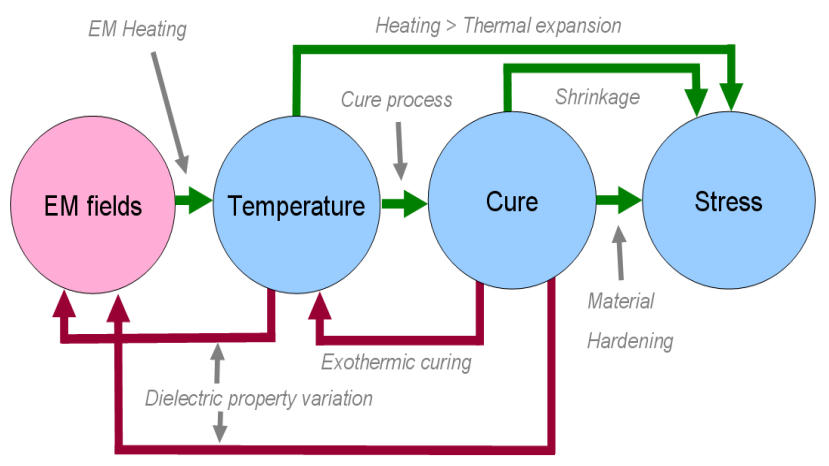

Figure 3 Complex interaction in microwave cure process

\section{Test Strategy}

Each of the two packages to be analysed have been considered in a simplified form, consisting of a 'silicon die' covered in a layer of encapsulant material, as illustrated in Figure 4. The dimensions of the packages are outlined in table I. A commercially available thermosetting epoxy material has been used for this assessment. Differential Scanning Calorimetry (DSC) data has been analysed to determine the cure behaviour and exothermal energy released by the material. The coefficients used in the implemented Arrhenius cure model have been fitted to the DSC data using a separate numerical integration scheme. This scheme considered a very large number of permutations of the coefficients, assessing fit between experimental and model data for each coefficient set. Optimal coefficients resulted in a fair agreement between experimental and model cure-time-temperature curves. A more detailed DSC analysis is required to more accurately assess cure behaviour and to enable the cure model coefficients to be determined in a more intelligent manner. The thermomechanical load material properties are detailed in Table II. Experimental assessment of dielectric properties has been performed [7] using an Agilent Technologies 85070E dielectric probe kit. Obtained results indicated that both the real and imaginary components of the complex permitivity decrease with degree of cure. In the implemented numerical model, these components have been approximated by the functions given in Equations 1 and 2.

$$
\begin{gathered}
\varepsilon_{r}=4.06+(0.0062(T-298))-\left(0.35 \alpha^{*}\right) \\
\varepsilon^{\prime \prime}=0.41+(0.0040(T-298))-\left(0.50 \alpha^{*}\right)
\end{gathered}
$$

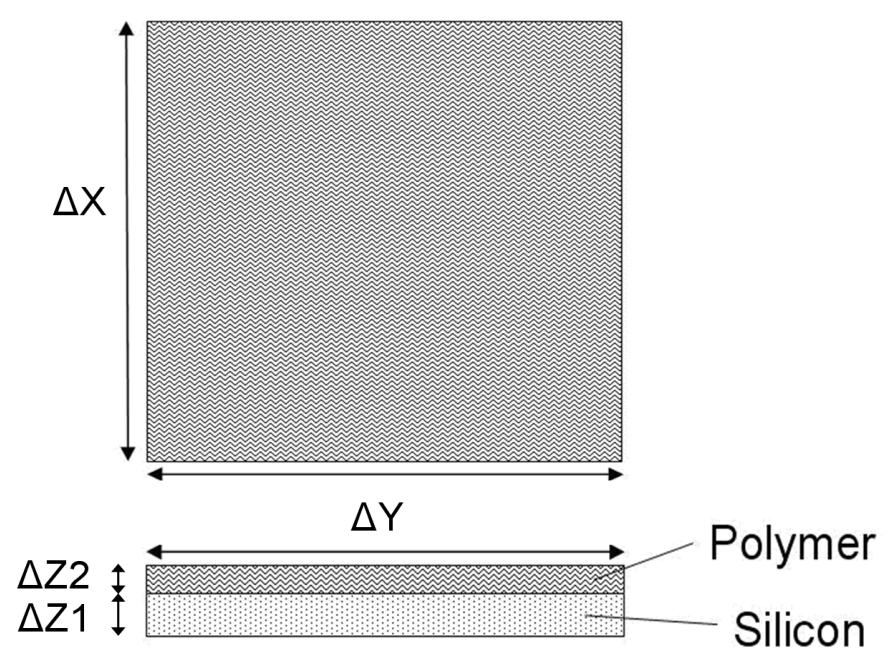

Figure 4 Package dimension schematic

Table I Package dimensions

\begin{tabular}{|c|c|c|c|}
\hline Symbol & Variable & $\begin{array}{c}\text { Package } \\
\# 1\end{array}$ & $\begin{array}{c}\text { Package } \\
\# 2\end{array}$ \\
\hline$\Delta \mathrm{X}$ & Package width & $7.5 \mathrm{~mm}$ & $22.5 \mathrm{~mm}$ \\
\hline$\Delta \mathrm{Y}$ & Package length & $7.5 \mathrm{~mm}$ & $22.5 \mathrm{~mm}$ \\
\hline$\Delta \mathrm{Z} 1$ & Silicon thickness & $2.0 \mathrm{~mm}$ & $4.0 \mathrm{~mm}$ \\
\hline$\Delta \mathrm{Z2}$ & Polymer thickness & $1.0 \mathrm{~mm}$ & $2.0 \mathrm{~mm}$ \\
\hline
\end{tabular}

Table II Load material properties

\begin{tabular}{|c|c|c|c|}
\hline Property & Silicon & Polymer & Unit \\
\hline Density & 2230 & 1100 & $\mathrm{~kg} \mathrm{~m}^{-3}$ \\
\hline Thermal conductivity & $\mathrm{f}(\mathrm{T})$ & 0.33 & $\mathrm{~W} \mathrm{M}^{-1} \mathrm{~K}^{-1}$ \\
\hline Specific heat & 970 & 700 & $\mathrm{~J} \mathrm{~kg}^{-1} \mathrm{~K}^{-1}$ \\
\hline Thermal expansion & $2.6 \mathrm{e}-6$ & $1.2 \mathrm{e}-4$ & $\mathrm{~K}^{-1}$ \\
\hline Young's modulus & 120 & $\mathrm{f}(\alpha)$ & $\mathrm{GPa}$. \\
\hline Poisson's ratio & 0.30 & 0.29 & - \\
\hline Dielectric constant & 12.0 & $\mathrm{f}(\mathrm{T})$ & $\mathrm{F} \mathrm{m}^{-1}$ \\
\hline Loss factor & 0.108 & $\mathrm{f}(\mathrm{T})$ & $\mathrm{H} \mathrm{m}^{-1}$ \\
\hline
\end{tabular}

\section{Results}


Packages \#1 and \#2 were considered to be subjected to the temperature profiles illustrated in Figure 5. Numerical analysis of the process was performed, with cure kinetics, temperature distribution and thermomechanical stress magnitude monitored continuously. The maximum and minimum degree of cure for each package is plotted in Figure 6. From these plots it is evident that the cure process for package \#1 is relatively rapid and results in a high maximal degree of cure with limited variation in degree of cure over the package. In contrast, package \# is not fully cured with a maximum degree of cure of approximately 0.8 and a wider variability in the cure over the package. Investigation of the numerical data suggests that the corners of the package are being insufficiently heated. This is likely to be due to the relative size of the package and the oven cross section. The fields vary sinusoidally across the cross section, with zero magnitude at the oven walls. There would therefore appear to be a requirement for the oven cross section to be greater than the package cross section. The relatively low degree of cure results in a lower Young's modulus within the polymer material. This is likely to be the cause of the disparity in the effective (von Mises) stress induced in the silicon die during the process as shown in Figure 7.

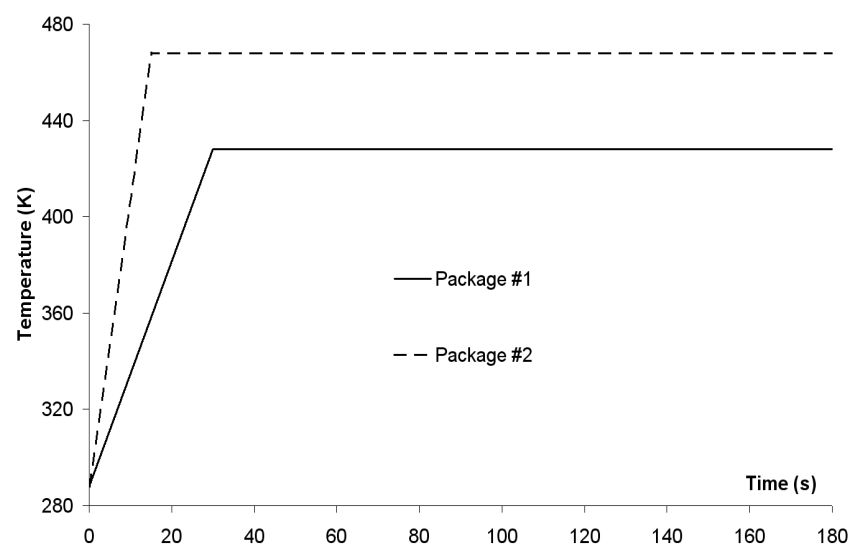

Figure 5 Temperature profiles for packages \#1 and \#2

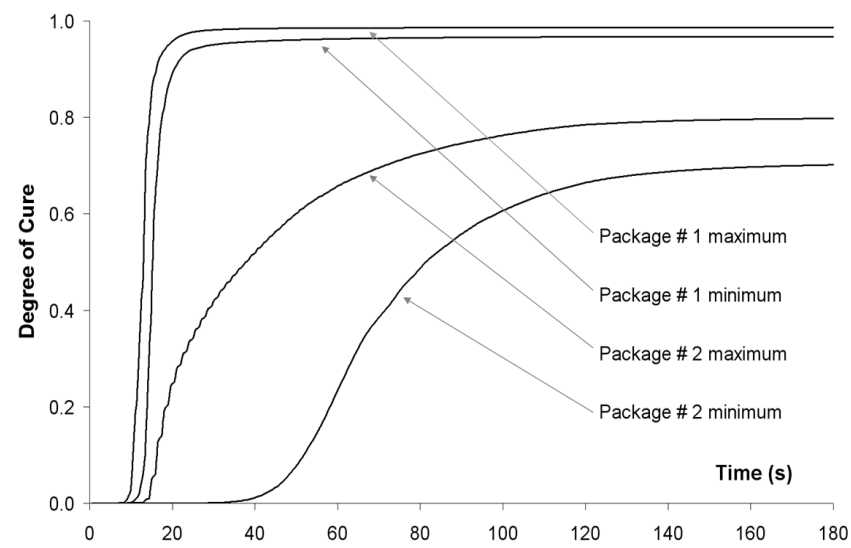

Figure 6 Evolution of degree of cure in packages \#1 and \#2 during a process time of $180 \mathrm{~s}$.

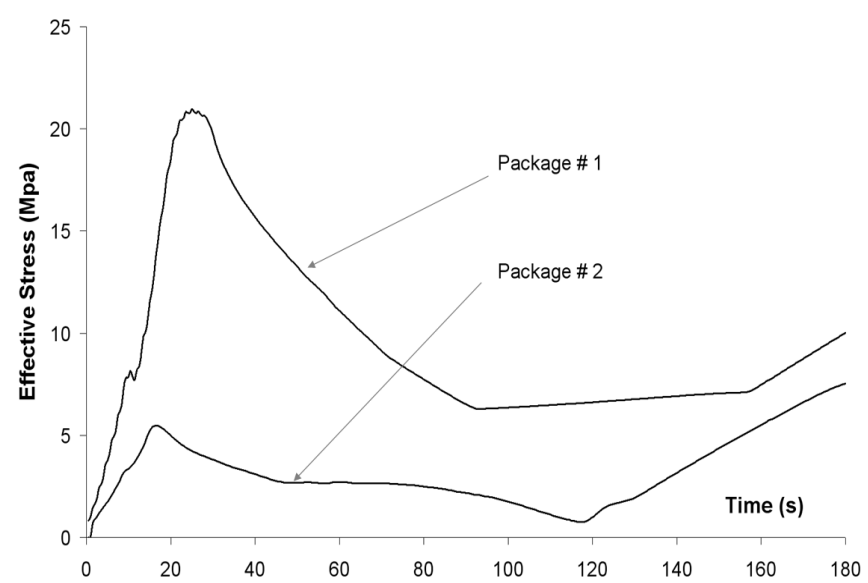

Figure 7 Evolution of effective stress in packages \#1 and \#2 during 180 second process time

\section{Conclusions}

Results obtained show that the VFM technology can be utilised to rapidly cure encapsulants in microelectronics applications. In cases in which the package is significantly smaller than the oven cross section, the degree of cure within the encapsulant is extremely high with little variation. However, issues are apparent when using a package with lateral dimensions comparable to those of the oven. This issue can be eliminated through adoption of a multi-oven tool head, which is capable of automatically swapping between oven units if differing dimensions.

Development of the FAMOBS system is continuing as part of a three-year project, supported through European Union 'Framework 7' funding. The project will focus on improvement of the system design to enhance performance and efficiency whilst decreasing cost. Materials used in the process will be characterised to determine their interaction with the microwave fields and the resulting cure process. New VFM-specific material may additionally be developed. Reliability of the VFM processed packages will be compared with conventionally cured packages through the use of a variety of accelerated lifetime testing approaches

Further information on this EU project can be found at the projects website: www.famobs.eu

\section{Acknowledgments}

The authors wish to acknowledge funding and support from the European Union Framework 7 programme (FP7SME-2007-2), contract number 218350. This project also benefits from the financial support of our partners, Eesti Innovatsiooni Instituut, Fraunhofer Gesellschaft (IPA), Camero di Commercio Industria, Artigianato e Agricoltura di Milano, Mikrosystemtechnik Baden-Württemberg e.V., the National Microelectronics Institute, the European Society for Precision Engineering and Nanotechnology, ACI-ecotec $\mathrm{GmbH} \&$ Co. KG, Kepar Electronica S.A., Satel S.A., Industrial Microwave Systems Ltd. and Ribler GmbH.

\section{References}

1. T. Tilford, K. I. Sinclair, G. Goussetis, C. Bailey, M. P. Y. Desmulliez, A. K. Parrott and A. J. Sangster, "Comparison of Encapsulant Curing with Convection and Microwave Systems," Proceedings 33rd International Electronics 
Manufacturing Technology Conference, Penang, Malaysia, 2008.

2. Sinclair, K. I., Desmulliez, M. P. Y. and Sangster, A. J., 2006, "A novel RF-curing technology for microelectronics and optoelectronics packaging," Proc. IEEE Electronics Systemintegration Technology Conference. 2006, Vol. 2, pp. 1149-1157.

3. Yee, K., 1966, "Numerical solution of initial boundary value problems involving Maxwell's equations in isotropic media,". IEEE Transactions on Antennas and Propagation, 14: PP. 302-307.

4. PHYSICA (1996-2007). Physica Ltd, 3 Rowan Drive, Witney, Oxon, United Kingdom, http://www.physica.co.uk.

5. T. Tilford, K. I. Sinclair, C. Bailey, M. P. Y. Desmulliez, G. Goussettis, A. K. Parrott and A. J. Sangster, "Multiphysics Simulation of Microwave Curing in MicroElectronics Packaging Applications," Journal of Soldering and Surface Mount Technology, Volume 19, Issue 3, 2007, pp. 26-33.

6. T. Tilford, E. Baginski, J. Kelder, A. K. Parrott and K. A. Pericleous, (2007) "Microwave Modelling and Validation in Food Thawing Applications," Journal of Microwave Power and Electromagnetic Energy, 41(4), pp. 30-45.

7. K. I. Sinclair, T. Tilford, G. Goussetis, C. Bailey, M. P. Y. Desmulliez, A. K. Parrott and A. J. Sangster, "Advanced Microwave Oven for Rapid Curing of Encapsulant," proceedings 2nd Electronics Systemintegration Technology Conference 2008, Greenwich, UK, pp. 551556, ISBN 978-1-4244-2813-7. 